\title{
Brood Parasitism Is Linked to Egg Pattern Diversity within and among Species of Australian Passerines
}

\author{
Iliana Medina, ${ }^{1, \star}$ Jolyon Troscianko, ${ }^{2}$ Martin Stevens, ${ }^{2}$ and Naomi E. Langmore ${ }^{1}$ \\ 1. Department of Ecology, Evolution, and Genetics, Australian National University, Canberra, Australia; 2. Centre for Ecology \\ and Conservation, College of Life and Environmental Sciences, University of Exeter, Cornwall TR10 9FE, United Kingdom
}

Submitted February 25, 2015; Accepted September 18, 2015; Electronically published February 3, 2016

Online enhancements: appendix. Dryad data: http://dx.doi.org/10.5061/dryad.s31sp.

\begin{abstract}
AвSTRACT: Bird eggs show striking diversity in color and pattern. One explanation for this is that interactions between avian brood parasites and their hosts drive egg phenotype evolution. Brood parasites lay their eggs in the nests of other species, their hosts. Many hosts defend their nests against parasitism by rejecting foreign eggs, which selects for parasite eggs that mimic those of the host. In theory, this may in turn select for changes in host egg phenotypes over time to facilitate discrimination of parasite eggs. Here, we test for the first time whether parasitism by brood parasites has led to increased divergence in egg phenotype among host species. Using Australian host and nonhost species and objective measures of egg color and pattern, we show that (i) hosts of brood parasites have higher within-species variation in egg pattern than nonhosts, supporting previous findings in other systems, and (ii) host species have diverged more in their egg patterns than nonhost species after controlling for divergence time. Overall, our results suggest that brood parasitism has played a significant role in the evolution of egg diversity and that these effects are evident, not only within species, but also among species.
\end{abstract}

Keywords: diversity, egg phenotype, brood parasitism, variability, diversity, Cuculidae.

\section{Introduction}

Explaining phenotypic diversity is one of the main challenges of evolutionary biology. To understand observed diversity patterns, it is important to identify the mechanisms that underlie phenotypic variation within species and then study these in a broader context with the use of phylogenetic methods. For instance, egg phenotype in birds is impressively diverse, and such variability is unevenly distributed across the avian phylogeny; some families of birds produce immaculate white eggs exclusively, whereas others show dramatic variability between and even within species (Kilner 2006). Earlier studies have proposed thermoregulation and camouflage as possible explanations for this vari-

\footnotetext{
* Corresponding author; e-mail: iliana.medina@anu.edu.au.
}

Am. Nat. 2016. Vol. 187, pp. 351-362. (C) 2016 by The University of Chicago. 0003-0147/2016/18703-56092\$15.00. All rights reserved. DOI: $10.1086 / 684627$ ability (Westmoreland et al. 2007; Mayer et al. 2009), but another interesting hypothesis that has not been explicitly tested is that coevolution between avian interspecific brood parasites and their hosts might drive increased diversity in egg phenotypes among species (Kilner 2006).

Avian brood parasites lay their eggs in the nests of other species (their hosts). This behavior has evolved independently seven times in the avian phylogeny, and the largest radiation has occurred in the family Cuculidae ( $\sim 40$ species; Payne and Payne 1998). Parasitism typically results in the loss of host young, and the host parents then invest many weeks rearing the imposter chick, often reducing opportunities for renesting within the season (Davies 2000). This behavior is so costly to the host that it has led to the evolution of multiple defenses against parasitism, including mobbing of adult brood parasites (Welbergen and Davies 2009; Feeney et al. 2012; Langmore et al. 2012) and rejection of foreign eggs (Rothstein 1975; Moksnes et al. 1991; Avilés et al. 2004; Spottiswoode and Stevens 2010), chicks (Grim 2007; Langmore et al. 2003; Sato et al. 2010), or fledglings (de Mársico et al. 2012).

Rejection of foreign eggs by hosts is one of the most widespread defenses against brood parasitism. In empirical studies, $63.3 \%$ of commonly exploited species showed egg rejection (i.e., they effectively reject $>90 \%$ of foreign eggs; Soler 2014). The evolution of egg rejection has led to an arms race; egg rejection by hosts selects for brood parasite eggs that resemble those of the host (Stoddard and Stevens 2010, 2011), which in turn selects for changes to the host egg phenotype that facilitate discrimination of foreign eggs (Davies 2000).

Brood parasitism has proved to be a particularly important driver of variation in egg phenotype within species (Stokke et al. 2002; Underwood and Sealy 2002; Kilner 2006; Spottiswoode and Stevens 2012). Host species can evolve different strategies to increase discrimination abilities and facilitate the detection of a parasitic egg. Theory predicts that (i) hosts should evolve eggs that are individually distinct from those of other females (e.g., high within-species variation), which decreases the likelihood that a parasite's eggs will 
match their own eggs, and (ii) hosts should evolve reduced within-clutch variation, because uniformity should facilitate discrimination of a foreign egg (Davies and Brooke 1989a; Langmore and Spottiswoode 2012). The first hypothesis has been well supported by comparing within-population egg variation in host populations that are allopatric and sympatric with brood parasites (Avilés and Møller 2003; Lahti 2005) and in comparative analyses (Soler and Møller 1995; Stokke et al. 2002). Moreover, individuals within the same host species may evolve distinctive signature patterns of blotches and markings (Swynnerton 1918; Victoria 1972; Stoddard et al. 2014; Caves et al. 2015), which makes their eggs highly recognizable. The second hypothesis has received mixed support. Some studies have found greater within-clutch egg uniformity in hosts than nonhosts (Avilés and Møller 2003; Moskát et al. 2008), but many others have failed to find support for the prediction (Avilés et al. 2004; Stokke et al. 2004; Cherry et al. 2007; Landstrom et al. 2010; Stoddard et al. 2014). Discrimination can be achieved by the use of just one of the strategies mentioned above; for example, species with high within-clutch variation can still have highly recognizable eggs if these have distinctive markings (Stoddard et al. 2014). Indeed, a recent study used image analysis and calculations of "entropy" of the egg markings of hosts and nonhosts of two African parasitic species, showing that host egg color and marking components have lower levels of corre- lation with one another than do those of nonhosts, thus potentially affording greater information about egg identity (Caves et al. 2015).

Many studies have supported the predictions stated above within various host species (Soler and Møller 1995; Stokke et al. 2002; Spottiswoode and Stevens 2011; Stoddard et al. 2014; Caves et al. 2015), suggesting that variation in egg color and pattern within species may be a response to brood parasitism. But can brood parasitism also be associated with egg diversity among species? If there is selection for novel phenotypes within host species (e.g., to have particularly distinguishable patterns, which facilitate discrimination of parasite eggs), and host eggs are selected to occupy different regions in the phenotypic space, variation among host species may arise as a byproduct of selection (fig. 1). The scenario described above leads to the prediction that, in the same amount of evolutionary time, two host species that are subject to brood parasitism will evolve more differences in egg phenotype between them than two nonhost species. Alternatively, increased constraints on the evolution of egg phenotype of hosts could result in decreased diversity of egg phenotypes among host species. The hypothesis that brood parasitism is associated with increased egg diversity among species is somewhat supported by an analysis performed at high taxonomic levels, where Kilner (2006) found a slight, nonsignificant association between high egg diversity

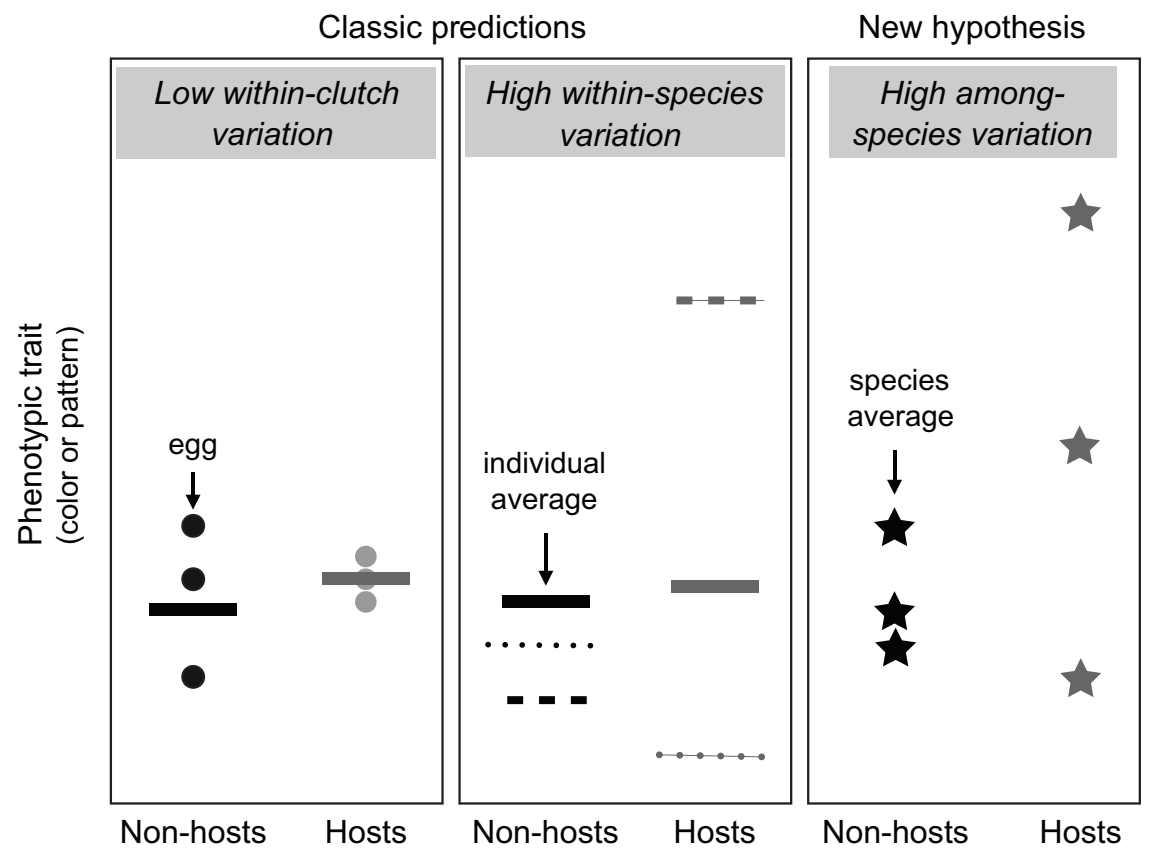

Figure 1: Illustration of the three hypotheses tested in the article. The $Y$-axis represents the values for any egg phenotypic trait (color or pattern). Left, points correspond to the value for each egg within a clutch. Middle, the different lines represent averages per individual in their egg phenotype. Right, the stars represent the average egg phenotype for a species, and hosts are predicted to have higher egg phenotypic variation among species than nonhosts. 
between species within a family and the level of exploitation by interspecific brood parasites. However, this study was based on family-level information and a data set that did not use objective measures of color and pattern. Since then, there have been well-resolved bird phylogenies and significant technological advances that allow quantification of color and pattern as seen through the eyes of a bird (Spottiswoode and Stevens 2010; Stoddard and Stevens 2011).

In our study, we use reflectance spectrometry, digital pattern analyses, and phylogenetic information to test whether brood parasitism is associated with higher egg diversity between host species. We used species of Australian hosts and nonhosts together with their phylogenetic relationships to test whether host species have evolved greater differences in egg phenotype than nonhost species, controlling for evolutionary time. Moreover, because our data set includes hosts of six different brood parasite species, we explored whether species exploited by the same parasite evolve more or fewer differences between them than host species parasitized by different species.

Australian brood parasites (cuckoos) and their hosts are a relatively unknown study system compared with those of Europe or North America, where the classic predictions associated with within-species variability have already been verified in comparative analyses (e.g., Soler and Møller 1995; Stokke et al. 2002). The diversity of hosts (main hosts $=90$ species) and cuckoo species $(n=10)$ breeding in Australia make this an ideal system for testing the two classic predictions associated with clutch variation; whether hosts have (i) low within-clutch variation and (ii) high within-species variation in egg morphology (fig. 1). We also use the Australian system to conduct the first test of the hypothesis that host species have evolved greater diversity in their egg phenotypes than closely related nonhosts (fig. 1). Eight of the 10 Australian parasitic cuckoos lay eggs that closely resemble those of their primary hosts (Brooker and Brooker 1989; Beruldsen 2003; Starling et al. 2006; Feeney et al. 2014), whereas two bronze-cuckoo species that parasitize domenesting hosts have evolved egg crypsis rather than mimicry (Langmore et al. 2009; Gloag et al. 2014). There is evidence of polymorphic, host-specific egg types in two cuckoo species, the Pallid cuckoo, Cacomantis pallidus, and the Brush cuckoo, Cacomantis variolosus (Beruldsen et al. 2003; Starling et al. 2006; Langmore et al. 2009). Like the majority of cuckoo hosts elsewhere, most cup-nesting host species in Australia show high rates of egg rejection (77\%; table A1, available online), suggesting that the morphology of host eggs may be under selection to facilitate accurate egg discrimination by hosts. Even among dome-nesting hosts, which show lower rates of egg rejection (12.66\%; table A1), egg morphology may be under selection as a result of brood parasitism, because the cuckoo removes a single egg during parasitism and is more likely to remove an egg with high luminance (Gloag et al. 2014), which may select for dark pigment in host eggs. Moreover, cuckoos in Australia are highly virulent, evicting all host eggs or outcompeting all host nestlings in the nest (Brooker and Brooker 1989). This results in higher costs of parasitism than for many hosts of nonevicting parasites (such as cowbirds Molothrus species and the greater spotted cuckoo Clamator glandarius), providing stronger selection for the evolution of defenses (Kilner 2005).

\section{Methods \\ Data Collection}

We took photographs and spectral data for 517 eggs in 200 clutches from 40 Australian passerine species (22 hosts and 18 nonhost species) belonging to 25 different genera (fig. A1; figs. A1-A4 available online) at the Australian National Wildlife Collection egg collection in Canberra (ACT). We measured three eggs per clutch (or fewer for species that lay smaller clutches) and five clutches per species, and we confirmed that the clutches were similarly distributed in time and space for hosts and nonhosts (fig. A2). Photographs were taken for egg pattern analyses, and spectral data were collected for color analyses (see below). For our analysis, we included some of the passerine species classified by Brooker and Brooker (1989) as either nonhosts $(n=18)$ or biological main hosts $(n=22)$ of Australian cuckoos. The species we used were selected from the Brooker and Brooker (1989) data set to maximize the number of phylogenetically independent lineages present in the sample while keeping a balance between the number of host and nonhost species. For instance, wherever there was a phylogenetically independent origin for host status (host or nonhost), we sampled that clade and its sister clade. Also, almost all of the thornbill and honeyeater species are parasitized; therefore, to avoid a bias in the analyses toward particularly large clades, we arbitrarily sampled four or five species as representatives of the clade. We collected data on host status from Brooker and Brooker (1989). They applied rigorous criteria to their classification of hosts as biological (successful) hosts, distinct from accidental or unsuitable hosts; biological hosts had multiple $(>4)$ independent $(>1$ observer, $>1$ location, $>1$ year) records of parasitism, and either (i) an egg or authentic nestling record followed through to fledgling; (ii) nestling and feeding records as well as egg records; or (iii) egg records alone, if congeneric with a known biological host of the cuckoo. We excluded species classified as nonbiological (occasional or rare) hosts, because it was not possible to infer the extent of selection on these hosts by brood parasites. We also collected data on nest type from the Handbook of the Birds of the World Alive (del Hoyo et al. 2014), given the evidence showing that egg rejection is less likely 
in enclosed nests than in open cup-shaped nests (Langmore et al. 2005). In our data set, $40 \%$ of the species were dome nesters.

\section{Egg Pattern Analyses}

We took photographs of each egg with a Canon EOS 50D camera and a 100-mm f/2.8 Macro lens. We included a $16 \%$ gray standard and a $1-\mathrm{cm}$ scale in each of the photographs. All image analyses were performed in ImageJ (Rasband 2014), using custom-written code. The green (medium wavelength) channel was extracted from each photograph and used for pattern analysis, following Spottiswoode and Stevens (2010). This approximates to an achromatic luminance channel of avian vision, with achromatic information widely thought to be most important in pattern processing in animal vision (Osorio and Vorobyev 2005). All photographs were taken in the same laboratory and under similar light conditions, but to further standardize for different lighting conditions, each image was also linearized (fit $R^{2}=0.999$ ) and converted to reflectance relative to the gray standard (Stevens et al. 2007). Images were scaled to 45 pixels per millimeter, and eggs were selected using an egg-shape selection tool (Troscianko 2014). Pattern analysis was then performed using fast Fourier transform bandpass filtering at different spatial scales from 2 pixels increasing exponentially with $\sqrt{2}$ to 512 pixels. This type of "granularity" pattern analysis has been used in a number of previous studies to analyze animal markings (e.g., Godfrey et al. 1987; Stoddard and Stevens 2010). The granularity filtering approach is broadly based on well-established principles of lower-level vision, including receptive fields and spatial frequency filtering, and is supported by the neurophysiology of a range of vertebrate and invertebrate animal species (Campbell and Robson 1968; Godfrey et al. 1987; Stoddard and Stevens 2010). In addition, granularity-based metrics have been tried and tested with several field experimental studies of egg rejection, showing that the pattern metrics derived do predict rejection behavior (e.g., Spottiswoode and Stevens 2010, 2012; Stevens et al. 2013). We therefore chose this method over a recent feature-detection-based approach (Stoddard et al. 2014), which used techniques developed for machine vision and object recognition, because there is little clear evidence that the latter approach approximates to how object and pattern recognition work in animals. In addition, the machine-learning approach is yet to be validated with behavioral egg-rejection experiments, and so we cannot at present say with confidence whether the measures derived from it are relevant to how birds reject foreign eggs.

The amount of "energy" contained in each pattern was measured using a histogram at each spatial scale as the standard deviation of the pixel intensities. The resulting granularity spectra were used to generate descriptive statistics of each egg pattern, including marking diversity (the proportion of the total energy accounted for by the dominant marking size; i.e., the higher the value, the more one marking size dominates), contrast of the patterns against the background (total energy or amplitude of the spectrum), and dominant marking size (denoted by peak frequency of the spectrum; Stoddard and Stevens 2010).

\section{Pattern Differences between Species}

Pattern difference between two species was calculated as the sum of the absolute differences in the species average energy at each spatial scale (i.e., the overall differences in the granularity spectra, taking into account both shape and amplitude). A matrix of between-species pairwise differences in pattern was generated. This measurement describes pattern similarity in a manner that, unlike the descriptive statistics, can compare multimodal energy spectra that have more than one peak frequency, preserving all pattern information and combining across all spatial scales measured.

\section{Pattern Variation within Species and within Clutches}

To calculate the degree of polymorphism within each species and within individuals, we used the mean contrast, mean dominant marking size, and mean marking diversity values from the pattern analysis for each photograph. We then calculated the standard deviation within each clutch (withinclutch variation) and used the average value per species. To calculate within-species variation in egg pattern, we calculated the standard deviation within each species using the same variables described above.

\section{Color Analyses}

We measured reflectance spectra of egg background color at three different places on the egg (base, middle, and tip) with a 5-mm-diameter probe and measured speckle color with a smaller probe (3-mm diameter) using an Ocean Optics USB4000 spectrometer. The visual systems of birds can be divided into two discrete classes; those with retinal pigments sensitive to shorter wavelength UV light (UVS group) and those with pigments sensitive to longer wavelength violet light (VS group; Cuthill 2006). Most passerines have UVStype cones, but shifts between visual systems can occur even within a single genus (Ödeen et al. 2012). Therefore, to assess egg color and pattern as seen through the eye of a bird, we calculated photon catches for the visual systems of both the blue tit (Cyanistes caeruleus) and the common peafowl (Pavo cristatus), which are commonly used models of a UVS and VS system, respectively (cone ratios UVS $1: 0.99: 0.71$ : 0.37 and VS $1: 1.9: 2.2: 2.1 ;$ Hart and Hunt 2007). Spectral 
sensitivity data are available for very few bird species (Hart 2001), so most studies use the visual systems of the blue tit Cyanistes caeruleus and the peafowl Pavo cristatus as models of UVS and VS visual systems, respectively (e.g., Avilés et al. 2010; Stoddard and Stevens 2010; Spottiswoode and Stevens 2012). These model species are distantly related, so they reveal the extent to which the results vary depending on the visual system used. We used a linear mixed model in $\mathrm{R}$ to compare the differences between visual systems; we report the $F$ statistic and the $P$ value.

Photon catch values were used in a model that predicts discrimination abilities for color and yields a value of just noticeable differences (JNDs). We used the log version of the standard Vorobyeb and Osorio model (1998) using Weber fractions of 0.05 or 0.02 where a JND of less than 1.00 means two objects are not distinguishable, and discrimination is unlikely when values are under 3 JNDs (Siddiqi et al. 2004). We generated a pairwise distance matrix of JNDs in color between all the species, for both visual systems. As in the case of pattern analysis, this pairwise approach is much more accurate than reducing variables to give color values per species. To calculate color polymorphism, we used the same procedures described in the pattern analyses using values for each cone.

\section{New Hypothesis: Diversity of Egg Color and Pattern among Species}

To test whether, on average, host species evolve more differences in egg phenotype than nonhost species, we made pairwise comparisons within two sets: among host species and among nonhost species. Phenotypic differences between species are expected to increase with time (Martins 1994); therefore, to make objective comparisons between host and nonhost pairs, we had to include information about the time of divergence between each pair of species. To do so, we downloaded 1,000 different possible phylogenetic trees from a pseudoposterior distribution from birdtree.org (Jetz et al. 2012). By doing the same analyses across different phylogenetic hypotheses, we made sure that our results were independent from the tree used. From each tree, we generated matrices with phylogenetic distances between species using the cophenetic.dist command in the $\mathrm{R}$ package ape (Paradis et al. 2004). Because the trees were time calibrated, distances are directly proportional to time (millions of years ago; MYA) and can actually be interpreted as such.

Because we were interested in finding out whether pairs of host species would diverge more than pairs of nonhost species, we included another variable called "type of comparison." This variable refers to the fact that some phenotypic distances were calculated between host species (host vs. host) and others were calculated between nonhost species (nonhost vs. nonhost). Thus, if hosts were more diverse, we would predict larger phenotypic distances in comparisons between host species than between nonhost species.

We used general linear models (Legendre and Fortin 2010) to test our hypothesis, and we included the following as predictors: phylogenetic distance, type of comparison, and phylogenetic distance $\times$ type of comparison. As response variables, we included egg color differences (JNDs) and egg pattern differences. This last variable was transformed using natural logarithm to achieve normality. We report the average $P$ value and average $\beta$ across the 1,000 trees for each predictor in the model. We also report the standard deviation for these values.

If phylogenetic distance predicts differences in color and pattern, it suggests that related species are more similar to each other than nonrelated species. If the type of comparison effectively predicts differences in color and pattern, it suggests that being a host or a nonhost species is associated with the degree of diversity in color and pattern, despite phylogenetic relatedness. If the interaction between both variables predicts differences in color and pattern, it suggests that color and pattern differences evolve differently in hosts and nonhosts (the slopes are different). Additionally, to explore whether host species that shared the same parasite evolved more or fewer phenotypic differences, we included a variable that coded whether the pair of host species shared the same parasite or a different parasite. It is worth pointing out that the rationale behind all this analysis is somewhat analogous to measuring evolutionary rates; if a pair $\mathrm{X}$ of species evolves more phenotypic differences than pair $\mathrm{Y}$ in the same time, it suggests that pair X evolved faster. We did not use specific methods to measure evolutionary rates for two reasons. First, current methods to measure pattern and color differences are much more accurate when doing pairwise comparisons than when summarizing information in a one-dimensional variable. Second, several nonhost species are contained within clades of hosts, and current phylogenetic methods are designed to calculate and compare rates between clades, not among singular branches.

\section{Classic Predictions: Variation in Egg Pattern within Species and within Clutches}

To test whether host eggs are more or less polymorphic than nonhosts, we used a phylogenetic linear regression (pgls) in the Caper package in R (Orme et al. 2012). We used host status and nest type as predictor variables and within-clutch and within-species variation in color and pattern as response variables. Nonsignificant predictors were dropped one at a time until we obtained a model with only significant predictors. We report the $P$ value, $\beta$, and $\lambda$ (e.g., phylogenetic signal, when $\lambda=0$ the relationship between predictor and response is unaffected by phylogeny). The analyses were repeated for 1,000 different trees obtained from birdtree.org 
(Jetz et al. 2012). The data set with final pattern and color measurements has been deposited in the Dryad Digital Repository, http://dx.doi.org/10.5061/dryad.s31sp (Medina et al. 2016).

\section{Results}

Eggs of both hosts and nonhosts varied from plain white eggs, such as those of some thornbills (Acanthiza species) and finches (Poephila species), to speckled and darker eggs, such those of fantails (Rhipidura species) and lyrebirds ( $\mathrm{Me}$ nura species). There were no significant differences in color or pattern between hosts and nonhosts (pgls, color: $P>.5$ for all the cones in both background and speckles; pattern: proportion energy $P=.40$, peak frequency $P=.18$, total energy $P=.23$ ). In our sample, we found no association between being a host and having a closed nest; the distribution of closed versus open nests was $40 \%$ for hosts and 38\% for nonhosts, and nest type had no significant effect in any of the analyses reported below.

\section{Differences between Visual Systems}

Differences in color (JNDs) between pairs of species were significantly higher when the data were analyzed with the blue tit visual system than with the peafowl system (fig. A3). This was true for both background (BG) and speckle color (SP; linear mixed model, BG: for host vs. host, $F=150.34$, $P<.0001, \mathrm{df}=228,459$; for nonhost vs. nonhost, $F=91.00$, $P<.0001, \mathrm{df}=180,303$; SP: for host vs. host, $F=244.14$, $P<.0001, \mathrm{df}=228,459$; for nonhost vs. nonhost, $F=$ 230.74, $P<.0001$, df $=180,303)$. The difference between the blue tit and peafowl visual system was more pronounced when comparing pairs of host species (host vs. host) than when comparing nonhosts $(F=150.34$ and $F=91.00$, respectively). This means that an animal with a blue tit-like visual system would detect even more color differences between host eggs than would an animal with a visual system like that of the peafowl. The same analyses were performed using a Weber fraction of 0.02 instead of 0.05 , and the results remain qualitatively the same. Despite the fact that the val- ues of JNDs are higher for the blue tit visual system, all other analyses shown below had the same qualitative results for both visual systems. Thus, from now on, we will refer only to the analyses employing the blue tit visual system and a Weber fraction of 0.05 .

\section{New Hypothesis: Diversity of Egg Color and Pattern among Species}

Overall, phylogenetic distance was a good predictor of differences in egg color and in egg pattern between species (table 1; fig. 2). However, phylogenetic distance had a weaker relationship with egg pattern differences between hosts than between nonhosts, as indicated by the significant differences in slope in figure 2. Moreover, in addition to the effect of phylogenetic distance, differences in egg pattern between host species were significantly higher than the differences between nonhost species. This means that, over the same period of evolutionary time, two host species will evolve more differences in egg pattern than two nonhost species. However, this was not true for color (table A1). There are no significant differences in JNDs for comparisons between hosts and nonhosts. All the findings described above remained the same after doing the analyses using 1,000 different phylogenetic trees. Results remained the same both after excluding species with closed nests and after excluding species with immaculate eggs (fig. A4). Furthermore, among hosts, pattern differences were significantly smaller between pairs of hosts that share the same parasite, after controlling for phylogenetic distance $(\beta=-14.55 \pm 3.71$, $P=.00016 \pm .0001$; fig. 3).

\section{Classic Predictions: Variation in Egg Pattern within Species and within Clutches}

Eggs of host species were significantly more polymorphic in egg pattern than were those of nonhost species (fig. 4). Specifically, differences in peak energy were significantly higher between individuals of host species (within-species variation), and differences in peak frequency are significantly higher within clutches of host species (within-clutch

Table 1: Mean statistics of egg phenotypic diversity between species

\begin{tabular}{|c|c|c|c|c|c|c|}
\hline \multirow[b]{2}{*}{ Predictor } & \multicolumn{2}{|c|}{ Differences in egg pattern } & \multicolumn{2}{|c|}{ JNDs in speckle color } & \multicolumn{2}{|c|}{ JNDs in background color } \\
\hline & $\beta$ & $P$ value & $\beta$ & $P$ value & $\beta$ & $P$ value \\
\hline Phylogenetic distance & $.003 \pm .0003$ & $.038 \pm .012$ & $.03 \pm .0005$ & $.031 \pm .02$ & $.012 \pm .001$ & $.0008 \pm .006$ \\
\hline Type of comparison & $.90 \pm .07$ & $.0006 \pm .0007$ & $.30 \pm .082$ & $.198 \pm .123$ & $.121 \pm .147$ & $.607 \pm .227$ \\
\hline $\begin{array}{c}\text { Phylogenetic distance } \times \\
\text { type of comparison }\end{array}$ & $.006 \pm .0007$ & $.013 \pm .010$ & $.007 \pm .0008$ & $.098 \pm .08$ & $.009 \pm .001$ & $.614 \pm .231$ \\
\hline
\end{tabular}

Note: We report the slope value and the significance for each predictor. Standard deviations were obtained from doing the analyses with 1,000 different phylogenetic hypotheses. JNDs = just noticeable differences. "Type of comparison" refers to whether the distance was calculated between two host species or two nonhost species. Significant $P$ values are in bold type. 


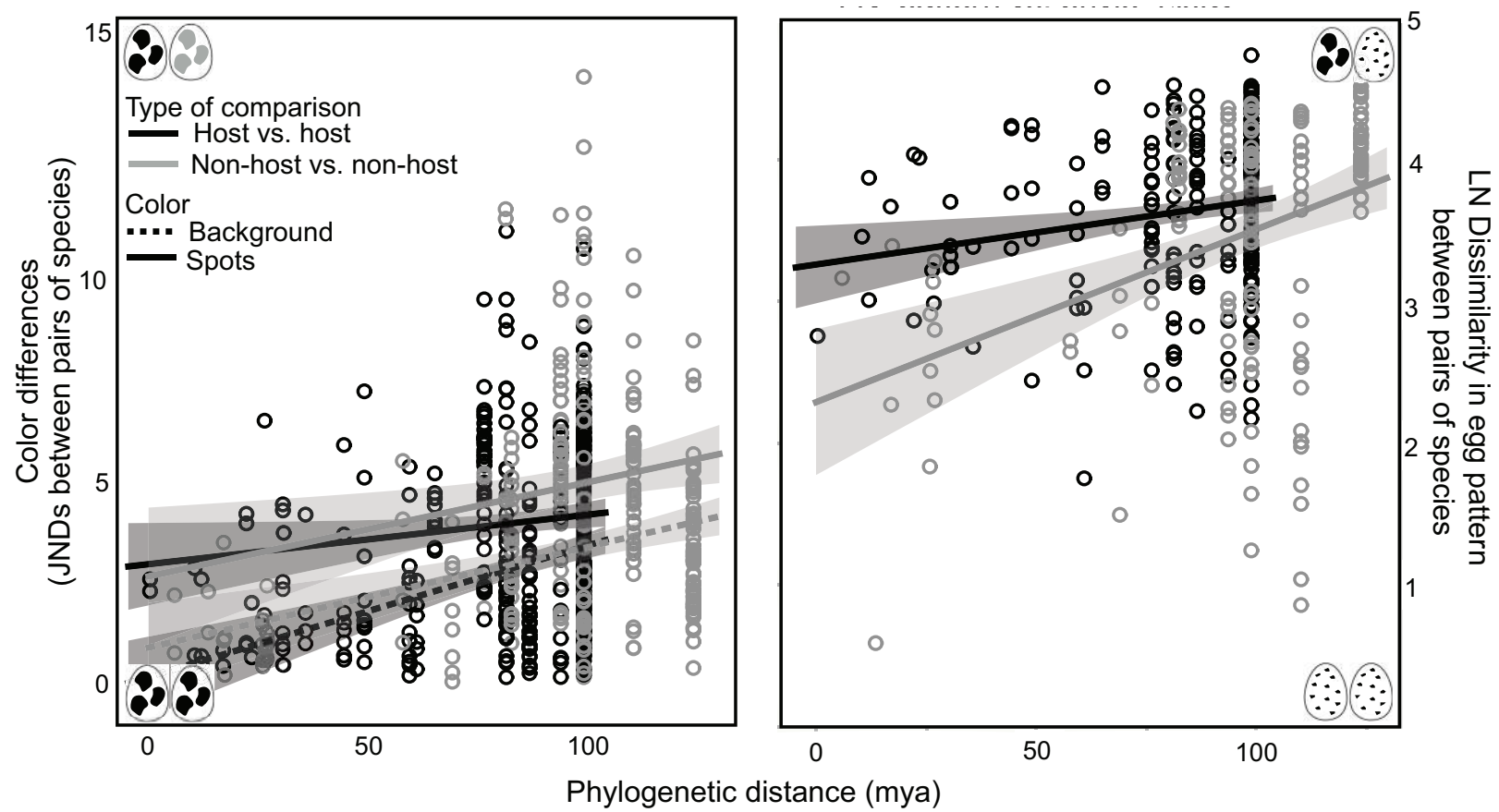

Figure 2: Phylogenetic distance explains differences between species in both egg coloration (left) and egg pattern (right). In the case of egg pattern, differences are significantly higher for host versus host comparisons (black) than for nonhost versus nonhost comparisons (gray). $\mathrm{JND}=$ just noticeable difference; $\mathrm{LN}=$ lognormal; mya $=$ million years ago.

variation). Differences remained significant after doing the analyses for 1,000 different phylogenetic trees (pgls, peak frequency \pm SD: $\lambda=0-0.762, \beta=28.81 \pm 13.980, P=$ $.0461 \pm .0001$; peak energy $\pm \mathrm{SD}: \lambda=0-0.438, \beta=$ $\left.2.22 \pm 1.057, P=.041 \pm 1 \times 10^{-4}\right)$. We found no evidence for differences in color variation or in total energy; hosts were no more polymorphic than nonhosts in egg background color $(\beta=-0.021, P=.251)$, speckle color $(\beta=-0.075$, $P=.483)$, or contrast $(\beta=3.25, P=.45)$.

\section{Discussion}

In this study, we aimed to explain avian egg diversity among species by testing the role of brood parasitism in the evolution of egg phenotype. By using a data set of Australian passerines that are hosts and nonhosts of different cuckoo species, we demonstrate that, in the same system, brood parasitism is associated with egg phenotypic variation within individuals, within species, and among species. We show for the first time, to our knowledge, that pairs of host species have diverged more in egg pattern than pairs of nonhost species. Additionally, divergence in egg phenotype is smaller in hosts that are exploited by the same parasite than in those exploited by different parasites.

Traditional hypotheses to explain egg phenotypic diversity include thermoregulation and camouflage against predation (Westmoreland et al. 2007; Mayer et al. 2009).
However, coevolution with brood parasites may also be responsible for phenotypic differences across species. Our analyses show that host species are likely to evolve more egg pattern differences among them than nonhost species, even if they have evolved independently for the same length of time. This supports a scenario where host species may escape a parasitic egg phenotype through the evolution of different strategies or different adaptive phenotypes, resulting in divergence. Our study also shows that egg pattern evolves differently in hosts and nonhosts. Under a neutral scenario, the expectation is that phenotypic differences between two species should increase in relation to the time since divergence (Martins 1994). This scenario is supported by the results for nonhost species (fig. 2). However, the slope of the relationship between phylogenetic distance and egg pattern dissimilarity is significantly less steep for the host versus host comparison (fig. 2). This suggests that phylogenetic history is less important than selective pressures in shaping the differences in egg pattern between host species. The results do not differ between open nesters and dome nesters (fig. A4), suggesting that possible egg rejection differences between hosts with different nest types are not critical at this macroevolutionary scale. Possible explanations for this are that (i) the effect is sufficiently strong to persist even with the inclusion of a few species with low rates of egg rejection, (ii) even low rates of egg rejection have evolutionary consequences, and (iii) the egg morphology of 


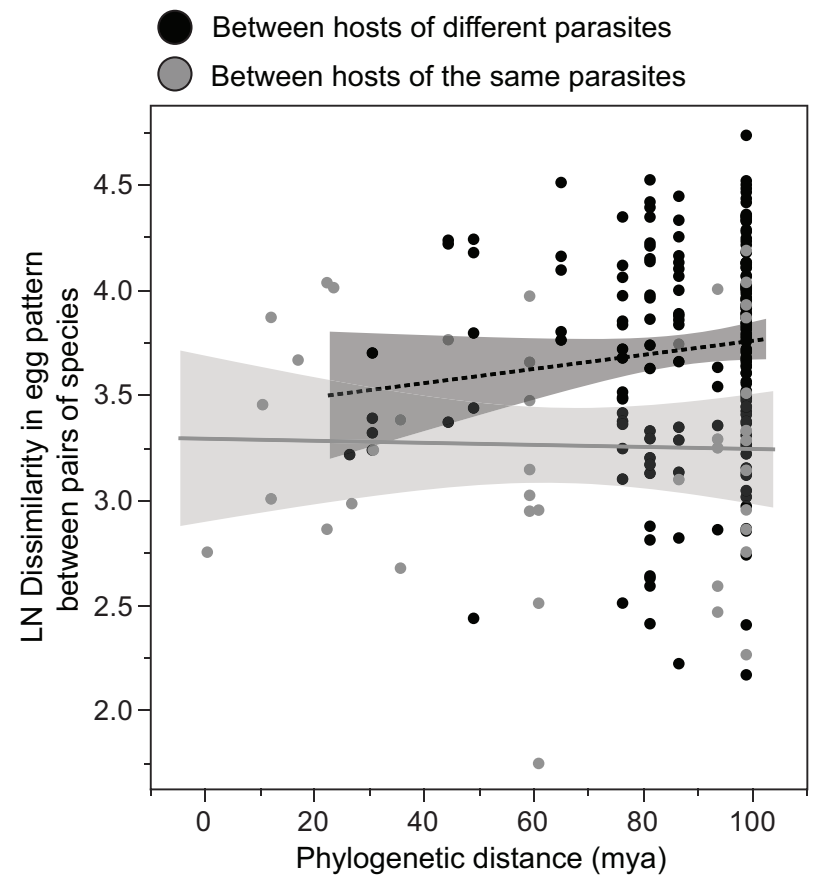

Figure 3: Levels of egg pattern dissimilarity are significantly higher between host species of different parasites (black) than between hosts of the same parasite species (gray). $\mathrm{LN}=$ lognormal; mya $=$ million years ago.

dome-nesting hosts may be under selection as a result of selective egg removal by the parasite (Gloag et al. 2014).

Our study shows that brood parasitism is associated with an increase in egg pattern divergence among host species and across different families. Moreover, we show that parasite diversity increases the likelihood of divergence. The egg pattern of hosts that are exploited by the same brood parasite was less diverse than that of hosts exploited by different parasites (fig. 3). This trend was to be expected, given that hosts of the same parasite are often phylogenetically related, so their eggs may be more similar from the outset, and they may be more likely to evolve along similar evolutionary trajectories.

We have shown that the eggs of host species have evolved more phenotypic differences than those of nonhost species, controlling for divergence time. Correspondingly, it is also possible that, in nonhost species, there are additional stabilizing selection sources on egg phenotype that hinder variability, such as camouflage and thermoregulation (StuartFox and Moussalli 2009; Vignieri et al. 2010; Hegna et al. 2013), whereas brood parasitism is the leading selective pressure in hosts.

We found no evidence to suggest that particular egg colors or patterns were associated with host status. Both immaculate and maculated eggs can be found in host and nonhost species. However, we found that host species were more variable in pattern than nonhost species. This supports a well-established hypothesis that a host's egg phenotype will evolve in random directions away from that of the parasite, thereby increasing within-species variation over time (Soler and Møller 1995; Lahti 2005; Spottiswoode and Stevens 2012). High variation in egg phenotype within species has been reported for hosts in comparative analyses done in other systems (Soler and Møller 1995; Kilner 2006). Moreover, eggs of European host species tend to be more polymorphic than those from North America, and this difference has been linked to the low specificity of the hostparasite system in the latter (Stokke et al. 2002). Our results also suggest that variation in the pattern, but not the color of the background or the speckles, is influenced by selection from brood parasitism, since we found no differences in variation between hosts and nonhosts for color traits. Our results correspond with studies of the European cuckoo, which show that pattern is an informative trait that increases likelihood of discrimination (Stoddard and Stevens 2010). Similarly, in passerines such as the village weaver Ploceus cucullatus and the bush warbler Cettia diphone, the presence of spots and their density are known to influence rejection probability (Higuchi 1998; Lahti and Lahti 2002).

Contrary to theoretical predictions, we found that withinclutch variation was also higher for hosts than nonhosts. Our study is the first comparative analysis to find this significant trend across different species. In theory, withinclutch variation should decrease in hosts, because this should facilitate discrimination between the host's own eggs and foreign eggs (Davies and Brooke 1989b; Jackson 1998). In support of this theory, reed warblers are more likely to reject foreign eggs if they lay a more uniform clutch (Stokke et al. 1999; Moskát et al. 2008). However, many empirical (Avilés et al. 2004; Cherry et al. 2007; Landstrom et al. 2010) and comparative analyses (Soler and Møller 1995; Stoddard et al. 2014) fail to support this hypothesis, and Cherry et al. (2007) found that, in a common cuckoo host, the great reed warbler (Acrocephalus arundinaceus), rejection of cuckoo eggs improved with increasing within-clutch variation (Cherry et al. 2007). Avilés et al. (2004) also reported that magpie hosts of the great-spotted cuckoo rejected more model eggs if the clutch was highly variable (Avilés et al. 2004). Stoddard et al. (2014) found that eggs could be easily recognizable in species with elevated levels of within-clutch variation if such variation is associated with having distinctive egg signatures (e.g., particular blotches and markings), such as in the brambling (Fringilla montifringilla). In Australian hosts, high within-clutch variation may also be related to the evolution of particular pattern signatures; however, this hypothesis remains to be tested.

Although the cause-effect relationship is hard to test, as in any other comparative analyses, three previous studies 
WITHIN CLUTCH Variation in egg pattern

(SD Peak frequency)

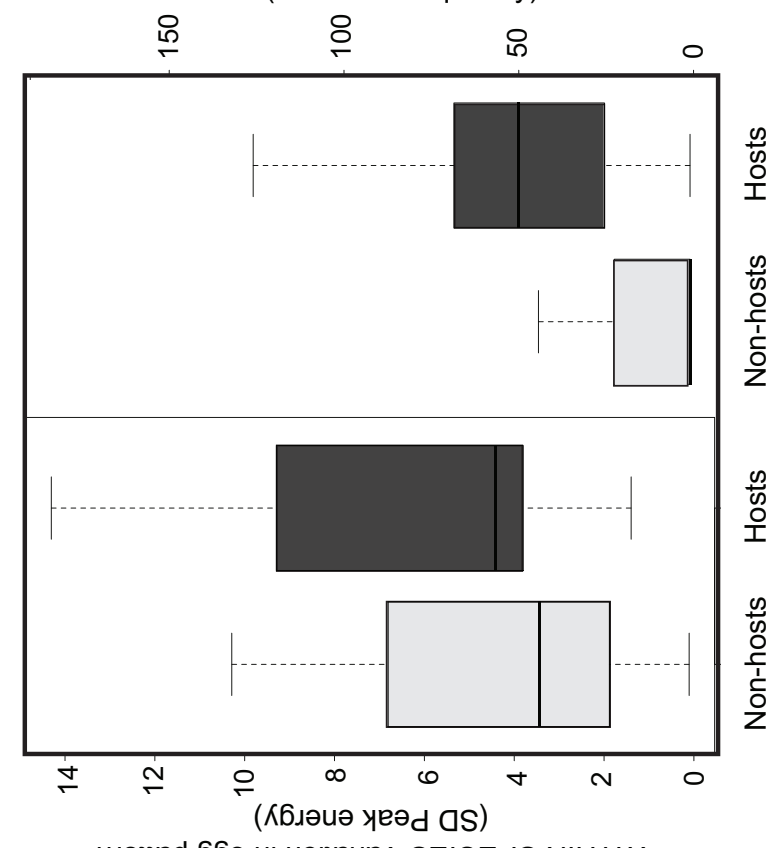

$m$

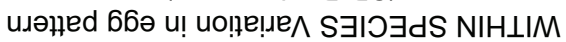

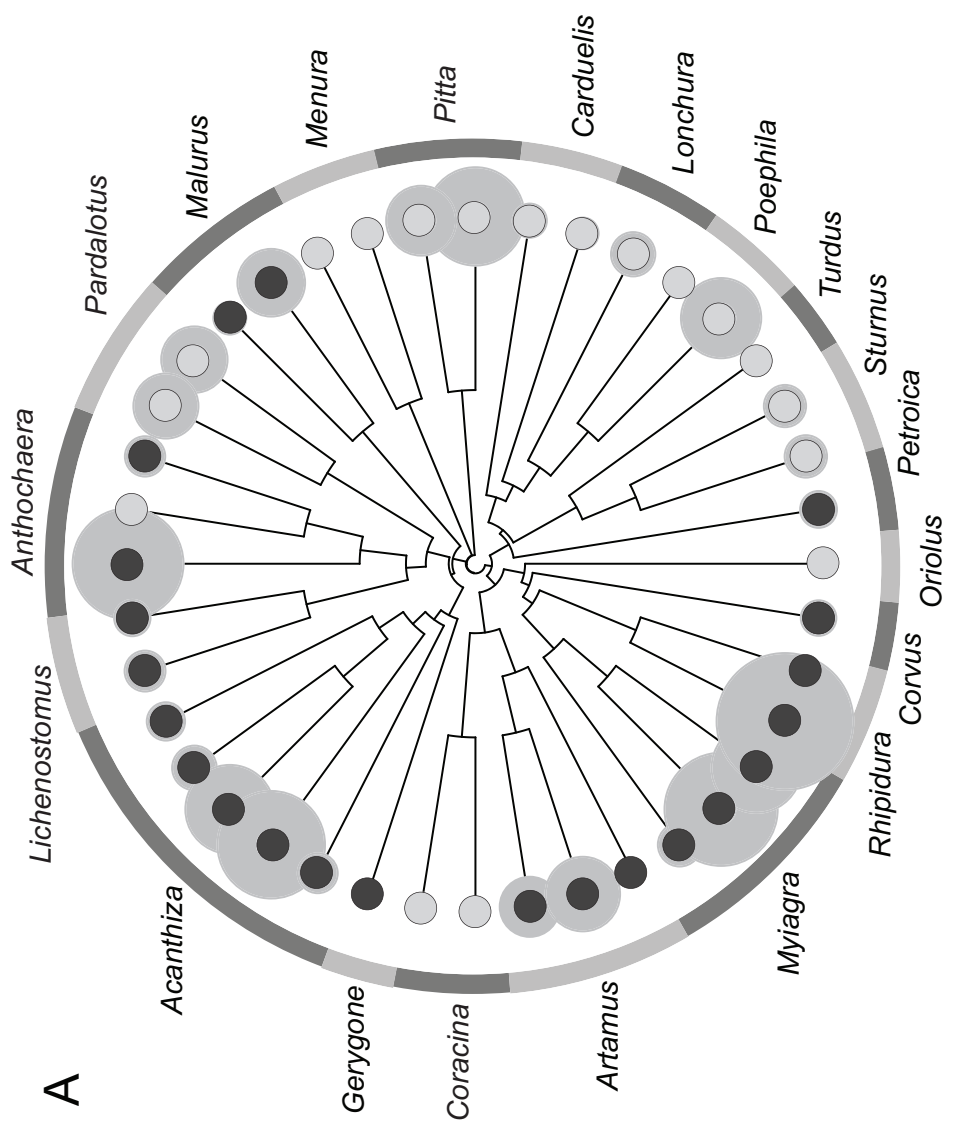

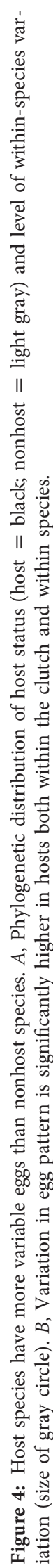

This content downloaded from 150.203.051.207 on March 23, 2016 17:11:28 PM All use subject to University of Chicago Press Terms and Conditions (http://www.journals.uchicago.edu/t-and-c). 
on the evolution of polymorphism in hosts strongly suggest that brood parasitism influences egg phenotype and not the other way around. Spottiswoode and Stevens (2012) showed that host species of brood parasites increase their egg variability after only 40 years of parasitism, and Lahti (2005) and Yang et al. (2014) demonstrated that egg variability is reduced when hosts are released from selection by brood parasites (Lahti 2005; Yang et al. 2014). Although we cannot completely reject the hypothesis that parasites selectively target hosts with high levels of variation, such a scenario seems implausible, because the evolution of egg mimicry in brood parasites would be constrained, leading to higher rates of rejection of brood parasite eggs.

Finally, we have shown that brood parasitism is associated with the generation of egg diversity at different taxonomic levels. Currently, there is no information on egg rejection rates for most of the species that we used in this study, but on the basis of our results, we predict high rates of egg rejection as a defense in these species. In fact, egg rejection has a high phylogenetic signal (Medina and Langmore 2015), and the average rejection levels for other Australian species from the same genera are high $(77.1 \%$ for open nesters and $12.66 \%$ for dome nesters; table A1). We would predict that similar systems of coevolution between hosts and highly virulent parasites, such as the European cuckoo, should also exhibit the evolutionary pattern that we report, because rejection rates are high in many European hosts (Langmore et al. 2005). Moreover, studying different components of egg phenotype allowed us to identify pattern as a more important trait than color. By using detailed data on numerous species, we were able to show that brood parasitism has deep implications for egg phenotype and is influential enough to leave traces at a larger evolutionary scale than previously studied.

\section{Acknowledgments}

We thank L. Joseph and R. Palmer for access to the Australian National Wildlife Collection egg collection and R. Vega, T. Wallenius, and two anonymous reviewers for very helpful comments on previous versions of the manuscript. I.M. was supported by an Australian National University Vice-Chancellor's travel grant, and N.E.L. was supported by the Australian Research Council.

\section{Literature Cited}

Avilés, J. M., and A. Møller. 2003. Meadow pitpit egg appearance in cuckoo sympatric and allopatric populations. Biological Journal of the Linnean Society 79:543-549.

Avilés, J. M., J. J. Soler, M. Soler, and A. P. Møller. 2004. Rejection of parasitic eggs in relation to egg appearance in magpies. Animal Behaviour 67:951-958.
Avilés, J. M., J. R. Vikan, F. Fossøy, A. Antonov, A. Moksnes, E. Røskaft, and B. G. Stokke. 2010. Avian colour perception predicts behavioural responses to experimental brood parasitism in chaffinches. Journal of Evolutionary Biology 23:293-301.

Beruldsen, G. 2003. Australian birds, their nests and eggs. Kenmore Hills, Queensland.

Brooker, M. G., and L. C. Brooker. 1989. Cuckoo hosts in Australia. Australian Zoological Reviews 2:1-67.

Campbell, F. W., and G. Robson. 1968. Application of Fourier analysis to the visibility of gratings. Journal of Physiology 197:551-566.

Caves, E., M. Stevens, E. Iversen, and C. Spottiswoode. 2015. Hosts of avian brood parasites have evolved egg signatures with elevated information content. Proceedings of the Royal Society B: Biological Sciences 282(1810). pii: 20150598.

Cherry, M. I., A. T. D. Bennett, and C. Moskat. 2007. Host intra-clutch variation, cuckoo egg matching and egg rejection by great reed warblers. Naturwissenschaften 94:441-447.

Cuthill, I. 2006. Mechanisms and measurement. Pages 3-40 in G. E. Hill and K. J. McGraw, eds. Bird coloration. Harvard University Press, Cambridge, MA.

Davies, N. B. 2000. Cuckoos, cowbirds and other cheats. Poyser, London.

Davies, N. B., and M. de L. Brooke. 1989a. An experimental study of co-evolution between the cuckoo, Cuculus canorus, and its hosts. I. Host egg discrimination. Journal of Animal Ecology 58:207-224.

- 1989b. An experimental study of co-evolution between the cuckoo, Cuculus canorus, and its hosts. II. Host egg markings, chick discrimination and general discussion. Journal of Animal Ecology 58:225-236.

del Hoyo, J., A. Elliott, J. Sargata, D. A. Christie, and E. de Juana. 2014. Handbook of the birds of the world alive. Lynx, Barcelona.

de Mársico, M. C., M. G. Gantchoff, and J. Reboreda. 2012. Hostparasite coevolution beyond the nestling stage? mimicry of host fledglings by the specialist screaming cowbird. Proceedings of the Roval Societv B: Biological Sciences 279:3401-3408.

Feeney, W. E., M. C. Stoddard, R. M. Kilner, and N. E. Langmore. 2014. "Jack of all trades" egg mimicry in the brood parasitic Horsfield's bronze-cuckoo. Behavioral Ecology 25:1365-1373.

Feeney, W. E., J. A. Welbergen, and N. E. Langmore. 2012. The frontline of avian brood parasite-host coevolution. Animal Behaviour 84: 3-12.

Gloag, R., L. Keller, and N. E. Langmore. 2014. Cryptic cuckoo eggs hide from competing cuckoos. Proceedings of the Roval Society B: Biological Sciences 281:1-7.

Godfrey, D. J., J. N. Lythgoe, and D. A. Rumball. 1987. Zebra stripes and tiger stripes: the spatial frequency distribution of the pattern compared to that of the background is significant in display and crypsis. Biological Journal of the Linnean Society 32:427-433.

Grim, T. 2007. Experimental evidence for chick discrimination without recognition in a brood parasite host. Proceedings of the Roval Societv B: Biological Sciences 274:373-381.

Hart, N. S. 2001. The visual evology of avian photoreceptors. Progress in Retinal and Eve Research 20:675-703.

Hart, N. S., and D. M. Hunt. 2007. Avian visual pigments: characteristics, spectral tuning and evolution. American Naturalist 169(suppl.): S7-S26.

Hegna, R. H., O. Nokelainen, J. R. Hegna, and J. Mappes. 2013. To quiver or to shiver: increased melanization benefits thermoregulation, but reduces warning signal efficacy in the wood tiger moth. Proceedings of the Roval Society B: Biological Sciences 280:1-9. 
Higuchi, H. 1998. Host use and egg color of Japanese cuckoos. Pages 80-93 in S. I. Rothstein and S. K. Robinson, eds. Parasitic birds and their hosts: studies in coevolution. Oxford University Press, New York.

Jackson, W. M. 1998. Egg discrimination and egg-color variability in the Northern Masked Weaver. Pages 407-416 in S. I. Rothstein and S. K. Robinson, eds. Parasitic birds and their hosts: studies in coevolution. Oxford University Press, Oxford.

Jetz, W., G. Thomas, A. Hartmann, and O. Mooers. 2012. The global diversity of birds in space and time. Nature 491:444-448.

Kilner, R. M. 2005. The evolution of virulence in brood parasites. Ornithological Science 4:55-64.

.2006. The evolution of egg color and patterning in birds. Biological Reviews 81:383-406.

Lahti, D. C. 2005. Evolution of bird eggs in the absence of cuckoo parasitism. Proceedings of the National Academy of Sciences of the USA 102:18057-18062.

Lahti, D. C., and A. R. Lahti. 2002. How precise is egg discrimination in weaverbirds? Animal Behaviour 62:1135-1142.

Landstrom, M. T., R. Heinsohn, and N. E. Langmore. 2010. Clutch variation and egg rejection in three hosts of the pallid cuckoo Cuculus pallidus. Behaviour 147:19-36.

Langmore, N., and R. Kilner. 2009. Why do Horsfield's bronze-cuckoo Chalcites basalis eggs mimic those of their hosts? Behavioral Ecology and Sociobiology 63:1127-1131.

Langmore, N. E., W. E. Feeney, J. Crowe-Riddell, H. Luan, K. M. Louwrens, and A. Cockburn. 2012. Learned recognition of brood parasitic cuckoos in the superb fairy-wren Malurus cyaneus. Behavioral Ecology 23:798-805.

Langmore, N. E., S. Hunt, and R. M. Kilner. 2003. Escalation of a coevolutionary arms race through host rejection of brood parasitic young. Nature 422:157-160.

Langmore, N. E., R. M. Kilner, S. H. M. Butchart, G. Maurer, N. B. Davies, A. Cockburn, N. A. Macgregor, et al. 2005. The evolution of egg rejection by cuckoo hosts in Australia and Europe. Behavioral Ecology 6:686-692.

Langmore, N. E., and C. N. Spottiswoode. 2012. Visual trickery in avian brood parasites. Pages 95-118 in D. Highes, J. Brodeur, and F. Thomas, eds. Host manipulation by parasites. Oxford University Press, Oxford.

Legendre, P., and M. J. Fortin. 2010. Comparison of the Mantel test and alternative approaches for detecting complex multivariate relationships in the spatial analysis of genetic data. Molecular Ecology Resources 10:813-844.

Martins, E. 1994. Estimating the rate of phenotypic evolution from comparative data. American Naturalist 144:193-209.

Mayer, P. M., N. G. Smith, R. G. Ford, D. C. Watterson, M. D. McCutchen, and M. R. Ryan. 2009. Nest construction by a groundnesting bird represents a potential trade-off between egg crypticity and thermoregulation. Oecologia 159:893-901.

Medina, I., J. Troscianko, M. Stevens, and N. E. Langmore. 2016. Data from: Brood parasitism is linked to egg pattern diversity within and among species of Australian passerines. American Naturalist, Dryad Digital Repository. http://dx.doi.org/10.5061/dryad.s31sp.

Medina, I. M., and N. E. Langmore. 2015. The costs of avian brood parasitism explain variation in egg rejection behaviour in hosts. Biology Letters 11:1-5.

Moksnes, A., E. Røskaft, and A. T. Braa. 1991. Rejection behavior by common cuckoo hosts towards artificial brood parasite eggs. Auk 108:348-354.
Moskát, C., J. M. Avilés, M. Ban, and A. Zölei. 2008. Experimental support for the use of egg uniformity in parasite egg discrimination by cuckoo hosts. Behavioral Ecology and Sociobiology 62:1885-1890.

Ödeen, A., S. Pruett-Jones, A. Driskell, J. K. Armenta, and O. Håstad. 2012. Multiple shifts between violet and ultraviolet vision in a family of passerine birds with associated changes in plumage coloration. Proceedings of the Roval Societv B: Biological Sciences 279: 1269-1276.

Orme, D., R. Freckleton, G. Thomas, T. Petzoldt, S. Fritz, N. Isaac, and W. Pearse. 2012. Caper: comparative analyses of phylogenetics and evolution in R. R package version 0.5 .

Osorio, D., and M. Voroyev. 2005. Photoreceptor spectral sensitivities in terrestrial animals: adaptations for luminance and colour vision. Proceedings of the Roval Society B: Biological Sciences 272:1745-1752.

Paradis, E., J. Claude, and K. Strimmer. 2004. APE: analyses of phylogenetics and evolution in R language. Bioinformatics 20:11.

Payne, R. B., and L. L. Payne. 1998. Parasitic birds and their hosts. Studies in Coevolution. Oxford University Press, Oxford.

Rasband, W. S. 2014. Image J. National Institutes of Health. http:// imagej.nih.gov/ij/.

Rothstein, S. I. 1975. Experimental and teleonomic investigation of avian brood parasitism. Condor 77:250-271

Sato, N. J., K. Tokue, R. A. Noske, O. K. Mikami, and K. Ueda. 2010. Evicting cuckoo nestlings from the nest: a new anti-parasitism behaviour. Biology Letters 6:67-69.

Siddiqi, A., T. Cronin, E. R. Loew, M. Vorobyev, and K. Summers. 2004 Interspecific and intraspecific views of color signals in the strawberry poison frog Dendrobates pumilio. Journal of Experimental Biology 207:2471-2485.

Soler, J. J., and A. Møller. 1995. A comparative analysis of the evolution of variation in appearance of eggs of European passerines in relation to brood parasitism. Behavioural Ecology 7:89-94.

Soler, M. 2014. Long-term coevolution between avian brood parasites and their hosts. Biological Reviews 89:688-704.

Spottiswoode, C. N., and M. Stevens. 2010. Visual modeling shows that avian host parents use multiple visual cues in rejecting parasitic eggs. Proceedings of the National Academy of Sciences of the USA 107:8672-8676.

- 2011. How to evade a coevolving brood parasite: egg discrimination versus egg variability as host defences. Proceedings of the Roval Societv B: Biological Sciences 278:3566-3573.

. 2012. Host-parasite arms races and rapid changes in bird egg appearance. American Naturalist 179:633-648.

Starling, M., R. Heinsohn, A. Cockburn, and N. E. Langmore. 2006 Cryptic gentes revealed in pallid cuckoos Cuculus pallidus using reflectance spectrophotometry. Proceedings of the Roval Society B: Biological Sciences 273:1929-1934.

Stevens, M., A. Parraga, I. Cuthill, C. Partridge, and T. Troscianko. 2007. Using digital photography to study animal coloration. Biological Journal of the Linnean Society 90:211-237.

Stevens, M., J. Troscianko, and C. N. Spottiswoode. 2013. Repeated targeting of the same hosts by a brood parasite compomises host egg rejection. Nature Communications 4:3-7.

Stoddard, M. C., R. M. Kilner, and C. Town. 2014. Pattern recognition algorithm reveals how birds evolve individual egg pattern signatures. Nature Communications 5:1-5.

Stoddard, M. C., and M. Stevens. 2010. Pattern mimicry of host eggs by the common cuckoo, as seen through a bird's eye. Proceedings of the Roval Society B: Biological Sciences 277:1387-1393. 
2011. Avian vision and the evolution of egg color mimicry in the common cuckoo. Evolution 65:2004-2013.

Stokke, B. G., A. Moksnes, and E. Røskaft. 2002. Obligate brood parasites as selective agents for evolution of egg appearance in passerine birds. Evolution 56:199-205.

Stokke, B. G., A. Moksnes, E. Røskaft, S. Rudolfsen, and M. Honza. 1999. Rejection of artificial cuckoo (Cuculus canorus) eggs in relation to variation in egg appearance among reed warblers (Acrocephalus scirpaceus). Proceedings of the Roval Societv B: Biological Sciences 266:1483-1488.

Stokke, B. G., G. Rudolfser, A. Moksnes, and E. Røskaft. 2004. Rejection of conspecific eggs in chaffinches: the effect of age and clutch characteristics. Ethology 110:459-470.

Stuart-Fox, D., and A. Moussalli. 2009. Camouflage, communication and thermoregulation: lessons from color changing organisms. Philosophical Transactions of the Roval Society B: Biological Sciences 364:463-470.

Swynnerton, C. F. M. 1918. Rejections by birds of eggs unlike their own: with remarks on some of the cuckoo problems. Ibis 60:127-154.

Troscianko, J. 2014. A simple tool for calculating egg shape, volume and surface area from digital images. Ibis 156:874-878.

Underwood, T. J., and S. G. Sealy. 2002. Adaptive significance of egg coloration. Pages $280-289$ in D. C. Deeming, ed. Avian incubation: behaviour, environment and evolution. Oxford University Press, Oxford.

Victoria, J. K. 1972. Clutch characteristics and egg discriminative ability of the African village weaver bird Ploceus cucullatus. Ibis 114: 367-376.

Vignieri, S., J. Larson, and H. Hoekstra. 2010. The selective advantage of crypsis in mice. Evolution 64:2153-2158.

Vorobyev, M., and D. Osorio. 1998. Receptor noise as a determinant of color thresholds. Proceedings of the Roval Society B: Biological Sciences 265:351-358.

Welbergen, J. A., and N. B. Davies. 2009. Strategic variation in mobbing as a front line of defense against brood parasitism. Current Biology 19:235-240.

Westmoreland, D., M. Schmitz, and K. E. Burns. 2007. Egg color as an adaptation for thermoregulation. Lournal of Field Ornithology 78:176-183.

Yang, C., Y. Liu, L. Zeng, and W. Liang. 2014. Egg color variation, but not egg rejection behavior, changes in a cuckoo host breeding in the absence of brood parasitism. Ecology and Evolution 4:22392246.

Associate Editor: Edmund D. Brodie III Editor: Yannis Michalakis 